\title{
How Long is the Worst Part of the COVID-19 Mortality Surge?
}

\author{
Valmor F. de Almeida ${ }^{a, *}$ \\ ${ }^{a}$ University of Massachusetts Lowell, Dept. of Chemical Engineering (Nuclear Program), Lowell, MA 01854
}

\section{ARTICLE INFO}

\section{Keywords:}

SARS-CoV-2

Mortality critical surge period

Virus pandemic

Data fitting

On-line data analytics

\begin{abstract}
A B S T R A C T
Objectives: Define and quantify the critical surge period of existing on-line COVID-19 mortality data; this could be instrumental to decision makers to determine how long health controls should be in place, and how long to expect the public health system to be in great stress. Create and share on-line, open-source software to continuously monitor critical surge periods in various communities as the data set grows. Test the critical surge period computational method on hierarchical data sets of various communities: a) globally; b) countrywide (states in the US); and c) statewide (counties/towns in the US states).

Study Design: This was an open-source, on-line, COVID-19 data analytics development with support for web-browsers on any current mobile device.

Methods: On-line, timely data for COVID-19 mortality was automatically retrieved by a developed application using the programming Python language and Jupyter electronic notebooks. An on-line, version-controlled code repository was created for the application and also used for providing an open execution environment for the research community. The data was fitted to a modified sigmoid function via an automated nonlinear least-squares method. Three optimal parameters were computed and the critical times for the maximum and minimum curvatures of the function were used to obtain the critical surge period. The goodness of the fit was measured by a standard coefficient of determination, and alternatively, by direct pointwise relative error.

Results: The software developed (a Python package) is called covid-surge (repository URL: htttps://github.com/dpploy/covid-surge) and available for download from PyPI on-line (URL: https://pypi.org/project/covid-surge/). Using covid-surge, the critical surge period was computed for the communities with the most evolved COVID-19 outbreak. At the time of this writing, the critical surge period was calculated for all countries with fully evolved mortality (12 out of 188) and found to be in average 23 days with 3 days of standard deviation. Similarly, for all states/districts in the US (20 out of 56), the computed average was 25 days with 3 days of standard deviation. Likewise, for the same states in the US, counties/towns that had fully evolved mortality surge (91 total out of 981 that had death cases) had the overall average of the surge period equal to $\mathbf{2 3}$ days with a standard deviation of 3 days. The accuracy of these results can be reproduced on-line using the software provided. In addition, forward results using newer data can also be generated by the research community using covid-surge.

Conclusions: The surge period for hierarchical data including 12 countries, 20 US states, and 91 US counties/towns is remarkably similar: a 24-day period with 3-day standard deviation for all communities across many countries and the US. All of these communities were under similar COVID-19 controls, including social distancing, improved hygiene, and isolation which helps explain the constancy of the surge period. That is, these communities experienced nearly the same amount of time during the most stressful period of the mortality surge. Although the extent to which epidemic controls are applied can vary from community to community, this study shows that the variability of the surge period is small. As the COVID-19 sweeps over the continents, additional data will be available for fully-evolved communities and a later comparison can be made with covid-surge on-line. This study suggests that communities not yet fully affected by the COVID-19 mortality may expect a period of high stress on their public health system of about 30 days (lower bound). That is, health care organizations should plan to operate at full capacity for a minimum of 30 days.
\end{abstract}

\footnotetext{
@valmor_dealmeida@uml.edu (V.F. de Almeida)

- www.uml.edu (V.F. de Almeida)

OrCID(s): 0000-0003-0899-695X (V.F. de Almeida)
} 


\section{Introduction}

A MONG many challenging decisions elected officials $A_{\text {face during the ongoing COVID-19 pandemic, one }}$ stands out, namely, deciding how long to enforce public health controls aimed at containing the virus. Specifically, isolation controls have a profound adverse impact on local and global economy therefore this is a matter of interest. While decisions cannot be made based on exact science alone, the scientific evaluation of emerging on-line mortality data from communities with fully evolved outbreaks can be used as a basis to establish how long the most stressful length of time typically lasts. This period is referred here as the mortality critical surge period, $\Delta T_{c}$, and defined below.

This short study, identified through data analysis, that diverse communities with fully evolved epidemics have essentially the same $\Delta T_{c}$. In this discovery process, an on-line computational utility was developed for gathering data automatically, compute $\Delta T_{c}$, and compare results for three hierarchical levels of mortality data, namely, globally (all countries), countrywide (states in the US), and statewide (county/towns in states of the US). For communities afflicted by the COVID-19 pandemic with sufficiently evolved transmission of the SARS-CoV-2 virus, the critical surge period is the number of days between the maximum and minimum curvatures points on the surge curve fitted by a modified sigmoid function:

$$
f(t)=\frac{\alpha_{0}}{1+\alpha_{1} e^{-\alpha_{2} t}},
$$

where $t$ is time, $f(t)$ is time-dependent cumulative mortality, i.e. number of accumulated deaths in the community, and the $\alpha_{i}, i=0,1,2$ are positive coefficients determined by data fitting. It can be shown that the critical surge period is simply

$$
\Delta T_{c}:=2 \tau \ln (2+\sqrt{3}),
$$

determined from the coefficient $\alpha_{2}=\tau^{-1}$ alone. By fitting the mortality data to $f(t)$, the optimal value of $\alpha_{2}$ is computed and therefore so is $\Delta T_{c}$. This interval, when it exists, can be unambiguously compared for data from different communities to shed light on the most stressful public health period. Therefore helping public officials to decide the length of time to enforce virus controls and make preparations for adequate health care capacity. Larger $\Delta T_{c}$ implies lesser stress imposed on the health system because there is more time for the system to adapt to the mortality surge. Therefore enforcement of public health controls for longer periods will increase $\Delta T_{c}$.

Not all mortality data will fit the modified sigmoid function so that all critical points can be identified. That is, communities with early epidemic evolution will not have sufficient data for the surge period to be computed. These cases are automatically found through the process of computing $\Delta T_{c}$ giving additional information on where these communities stand within the epidemic evolution.

\section{How long is the worst part of the mortality surge?}

To calculate $\Delta T_{c}$ for various data sets a computer program was developed [1] to automatically retrieve live data from on-line repositories. Specifically, the repository used in this study was from the John Hopkins University, URL: https://github.com/CSSEGISandData/ COVID-19/tree/master/csse_covid_19_data.

The computation of $\Delta T_{c}$ for 188 countries resulted in 12 countries (fig. 1): Belgium (19), France (19), Germany (21), Turkey (22), Spain (22), United Kingdom (23), Canada (23), Netherlands (23), Sweden (25), US (26), Italy (27), and Iran (30), with fully evolved epidemic. The numbers in parenthesis are the computed $\Delta T_{c}$ in number of days. All other countries either did not meet the condition of having a cumulative number of deaths equal to 2500 or more, or had no sufficiently evolved epidemics (with the exception of China's mortality data which was not used because of lack of consistency). The countries in the latter case were: Brazil, Mexico, India, Peru, Ecuador, and Russia, in order of increasing evolution; Brazil being the least evolved. Therefore the world average $\Delta T_{c}$ is $\mathbf{2 3}$ days with 3 days of standard deviation.

The computation of $\Delta T_{c}$ for all 56 states and districts of the US resulted in 20 states (fig. 2): Virginia (19), New York (20), Massachusetts (21), Connecticut (21), Michigan (22), Minnesota (23), New Jersey (23), Maryland (24), Louisiana (24), North Carolina (25), Indiana (26), Georgia (26), Florida (27), Ohio (27), Colorado (27), California (27), Illinois (28), Missouri (29), Washington (29) with fully evolved epidemic. All other states and districts did not meet one or more of the following conditions: a) number of $\mathrm{cu}-$ mulative deaths equal to or greater than 500; b) number of deaths per 100,000 people per year equal to or greater than 41; and c) not fully evolved epidemic. The value in $b$ ) is the number of deaths in US per 100,000 people per year reported in 2019 for chronic lower respiratory diseases. The reason to enforce $b$ ) is to select COVID-19 data that is relevant when compared to deaths caused by other respiratory diseases. The states not fully evolved in epidemics that met conditions a), b) and c) were: Arizona and Mississippi; Arizona being the least evolved. Therefore the US statewide average for the critical surge period is $\mathbf{2 5}$ days with 3 days of standard deviation.

The final evaluation of on-line data was applied to the 20 US states with fully evolved epidemics. That is, all available data for counties/towns for each state were used to compute their $\Delta T_{c}$. The exact same parameters used for the calculation of $\Delta T_{c}$ for states were also used 


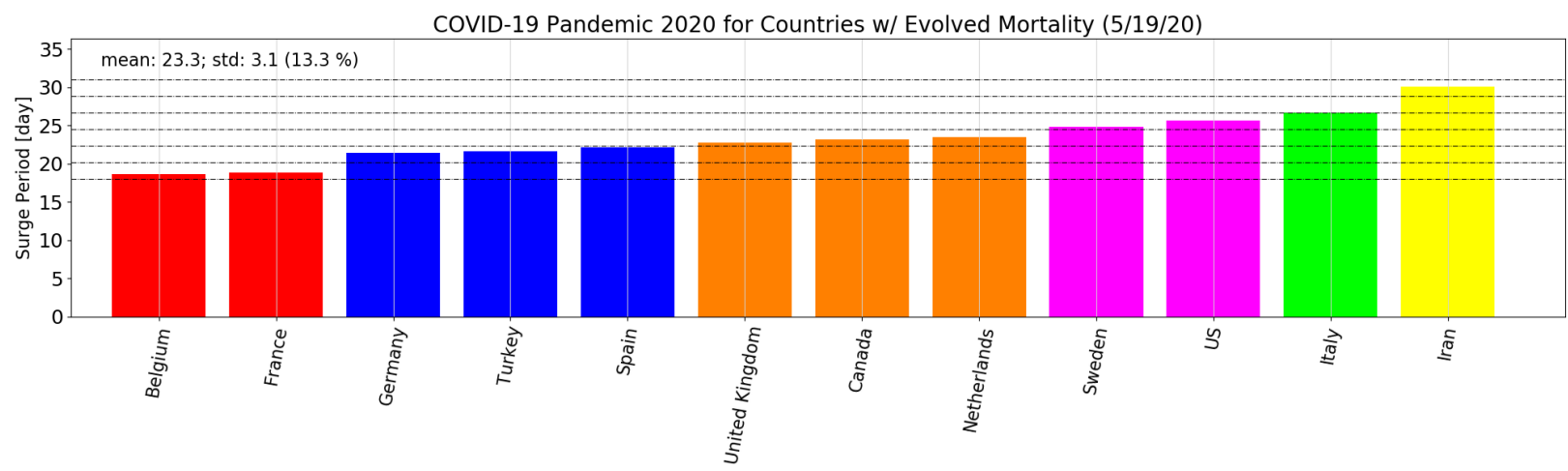

Figure 1: Global critical surge periods for all countries with fully evolved mortality.

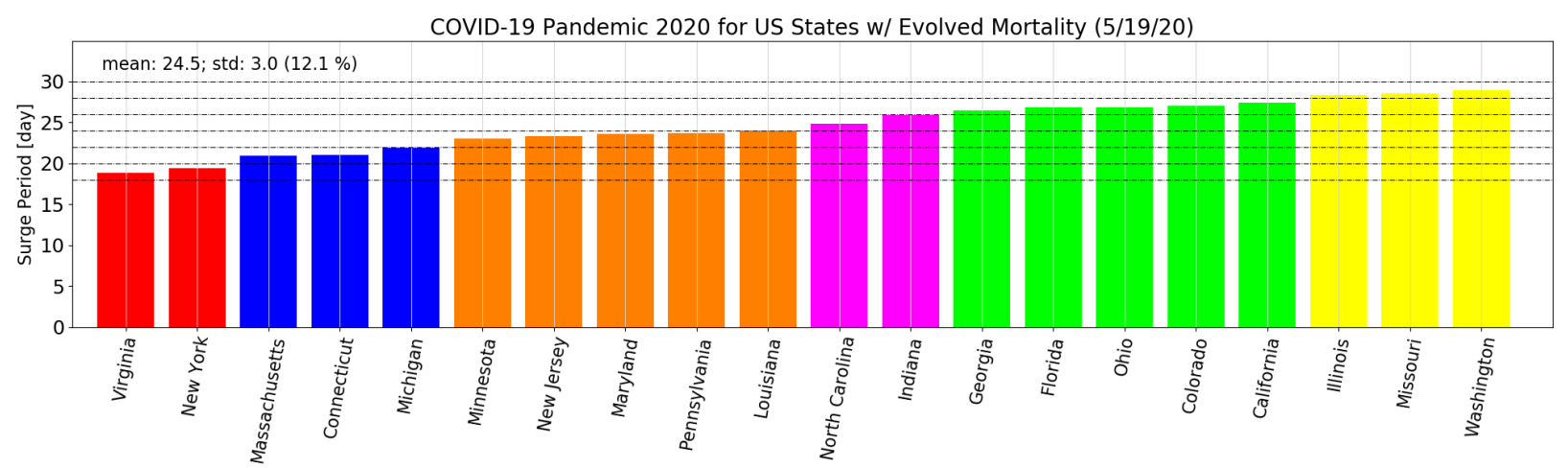

Figure 2: US critical surge periods for all states with fully evolved mortality.

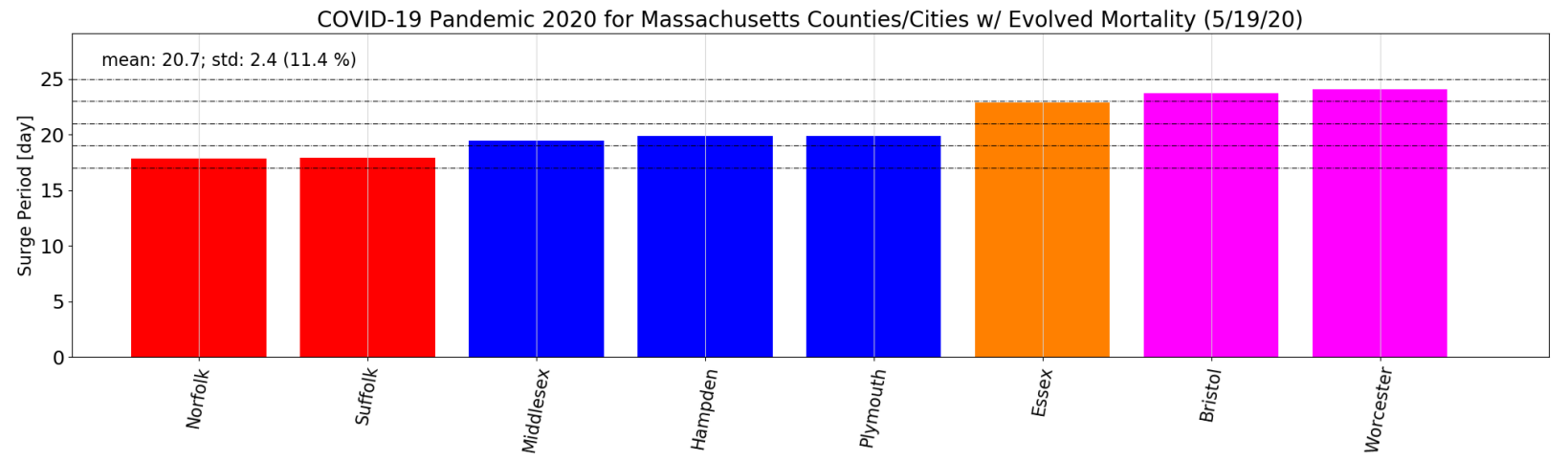

Figure 3: US critical surge periods for all Massachusetts counties/towns with fully evolved mortality.

here. A total of 981 counties/towns had death cases and 91 of those counties/towns (e.g. Massachusetts fig. 3) had fully evolved epidemics, therefore the critical surge period was computed for an average of $\mathbf{2 3}$ days and a standard deviation of 3 days. For instance, the state of Colorado had 5 counties/towns with evolved epidemics, Weld (24), Arapahoe (26), Denver (27), Adams (28), and Jefferson (32), with respective $\Delta T_{c}$ in days. 


\section{Conclusions}

The comparison of COVID-19 critical surge periods for hierarchical data shows that the average value is surprisingly constant across various levels of communities. The average value is $\mathbf{2 4}$ days with a standard deviation of $\mathbf{3}$ days. Therefore a basis of a 30-day period is the expected length of time to be the most stressful to the public health system. This period does not include the stresses caused by the incubation period of the epidemic where the surge is rising to the first critical point of the curve. Nevertheless, this observed 30-day period is an instructive length of time for public health officials to plan for the worst part of the surge. The live results presented here are reproducible and available to the research community through the on-line covid-surge application [1].

\section{References}

[1] de Almeida, V.F., 2020. Covid-surge: COVID19 mortality surge period calculation for communities afflicted by the corona virus SARS-CoV-2. http://pypi.org/project/covid-surge/. Python package. 
Graphical Abstract

How Long is the Worst Part of the COVID-19 Mortality Surge?

Valmor F. de Almeida

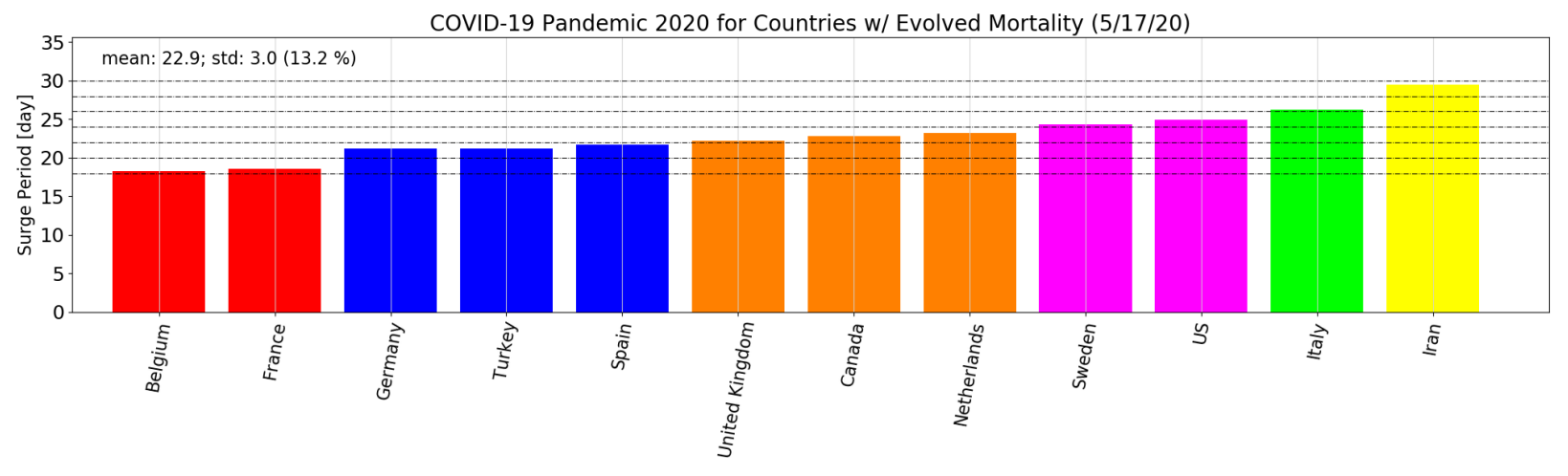


Highlights

\section{How Long is the Worst Part of the COVID-19 Mortality Surge?}

Valmor F. de Almeida

- A new indicator to quantify how long the worst part of the COVID-19 mortality surge lasts.

- An on-line, open-source computational tool for evaluating live data and present results to the public.

- Computed results of critical surge periods for data obtained at three levels: a) globally, b) at the US countrywide level, and c) at the US statewide level, to help public health officials to define the length of time to enforce epidemic controls. 